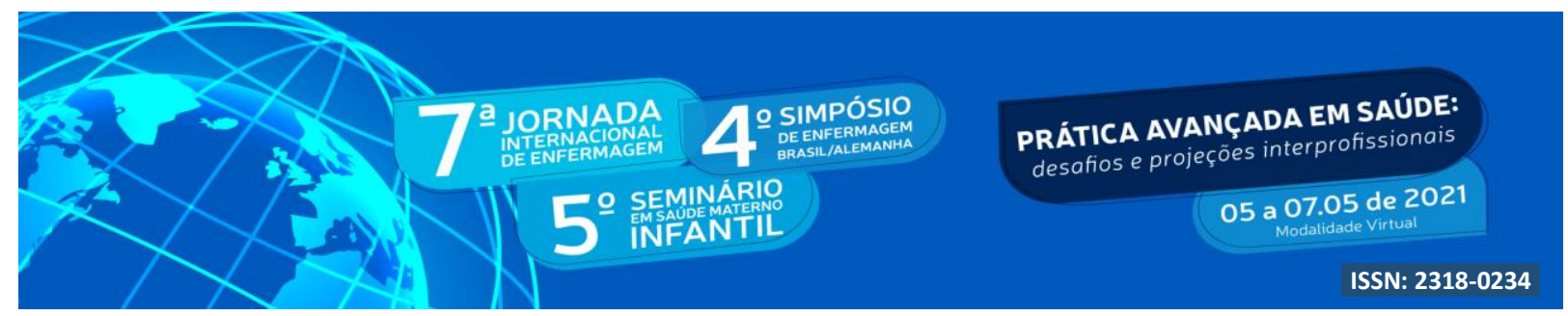

DOI: http://doi.org/10.48195/jie2021-087

\title{
QUALIFICAÇ̃̃O SOBRE SÍFILIS GESTACIONAL E CONGÊNITA PARA TRABALHADORES DE UMA UNIDADE DE SAÚDE ${ }^{1}$
}

\author{
Adriany da Rocha Pimentão²; Dedabrio Marques Gama ${ }^{3}$; Marcelo Nunes da Silva \\ Fernandes $^{4}$; Daiana Cristina Wickert ${ }^{5}$; Maria Denise Schimith ${ }^{6}$; Laís Mara Caetano da \\ Silva $^{7}$
}

RESUMO

Relato de um trabalho com o objetivo de qualificar os trabalhadores de uma Unidade de Saúde de um município do interior da Rio Grande do Sul sobre sífilis gestacional e congênita. Trata-se de uma proposta de intervenção a ser realizada com os trabalhadores de uma Unidade de Saúde. Para isso, propõem-se a realização de dois encontros com os trabalhadores, abordando-se temáticas relacionadas ao fluxograma de acompanhamento dos casos de sífilis gestacional e congênita e a elaboração de um protocolo municipal sobre o tema. Espera-se que a intervenção contribua para a qualificação da assistência à saúde da mulher, por meio da reflexão da equipe de saúde sobre a temática da sífilis. Soma-se a isso, a possibilidade de aperfeiçoar o processo de trabalho e de educação na saúde, por meio da melhoria do serviço de saúde às gestantes.

Palavras-chave: Educação; Sífilis congênita; Atenção Primária à Saúde.

\begin{abstract}
Report of a study with the objective of qualifying the workers of a Health Unit in a city in the interior of Rio Grande do Sul on gestational and congenital syphilis. It is an intervention proposal to be carried out with the workers of a Health Unit. For this purpose, it is proposed to hold two meetings with the workers, addressing issues related to the flowchart for monitoring cases of gestational syphilis and disease and the elaboration of a municipal protocol on the topic. It is expected that the intervention will contribute to the qualification of women's health care, through the reflection of the health team on the theme of syphilis. Added to this, the possibility of improving the work process and health education, through the improvement of the health service for pregnant women.
\end{abstract}

Keywords: Education; Congenital syphilis; Primary Health Care.

\footnotetext{
${ }^{1}$ Estudo de intervenção.

${ }^{2}$ Enfermeira. Mestranda em Enfermagem - PPGEnf/UFSM. adry.pimentao@gmail.com

${ }^{3}$ Enfermeiro. Mestrando em Enfermagem - PPGEnf/UFSM. dedabrio.gama@ gmail.com

${ }^{4}$ Enfermeiro. Doutorando em Enfermagem - PPGEnf/UFRGS. marcelonsf@ gmail.com

${ }^{5}$ Enfermeira. Mestranda em Enfermagem - PPGEnf/UFSM. daianacristinaw@ gmail.com

${ }^{6}$ Enfermeira. Professora do Curso de Enfermagem - UFSM. ma.denise2011@gmail.com

${ }^{7}$ Enfermeira. Orientadora. Professora do Curso de Enfermagem - UFSM. lais.silva@ufsm.br
} 


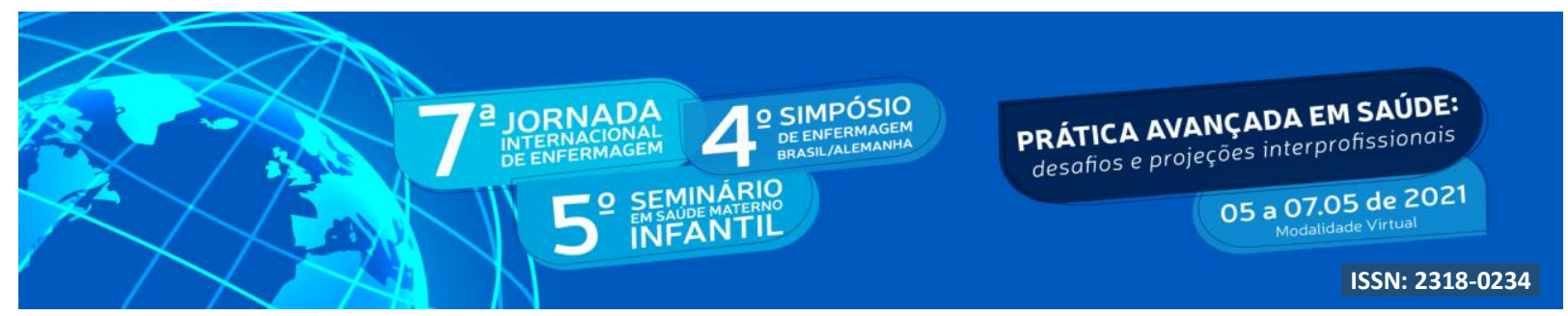

\section{INTRODUÇÃO}

As ações da Política Estadual de Saúde da Mulher têm foco na intersetorialidade da atenção integral à saúde da mulher, incluindo o planejamento, a qualificação e a implementação da rede de serviços a partir da Atenção Primária à Saúde (APS), passando pela Rede Cegonha e garantindo acesso ao atendimento de saúde às famílias, bebês, gestantes, com orientações e informações sobre planejamento familiar, saúde sexual e reprodutiva e redução da mortalidade materna, entre outras (BRASIL, 2004). Os principais problemas relacionados ao contexto da Rede Cegonha são os altos índices de sífilis na gestação e sífilis congênita, a falta de referência e contrarreferência, a falta de educação permanente em saúde (EPS) e a falta de materiais e equipamentos.

A sífilis é uma doença infectocontagiosa causada pela bactéria gram-negativa em forma de espiroqueta denominada Treponema pallidum (DE LORENZI et al., 2009). A sífilis congênita caracteriza-se pela transmissão do Treponema pallidum da gestante infectada não tratada, tratada inadequadamente ou reinfectada devido à falta de adesão do parceiro ao tratamento, para o concepto por via transplacentária, podendo ser transmitida em qualquer fase gestacional ou estágio da doença materna (VASCONCELOS, 2016).

No Brasil, houve um aumento progressivo na taxa de incidência de sífilis congênita, sendo que em 2004 a taxa era de 1,7 casos para cada 1.000 nascidos vivos e em 2013 subiu para 4,7 (VASCONCELOS, 2016). O aumento dos casos de sífilis associa-se, entre outras causas, a má qualidade do pré-natal e a falta de protocolos, uma vez que por meio do diagnóstico precoce, as gestantes infectadas podem ser tratadas (DAMASCENO et al, 2014). Entre os fatores de risco para a sífilis congênita destacam-se a não realização do pré-natal, o uso de drogas ilícitas pela gestante ou parceiro, a ausência de parceiro sexual fixo, o baixo nível socioeconômico, a baixa escolaridade, o acesso limitado aos serviços de saúde etc. (DAMASCENO et al., 2014).

O Ministério da Saúde (MS) recomenda que o rastreamento da sífilis durante o prénatal deve ser realizado na primeira consulta, durante o primeiro trimestre, e no terceiro trimestre da gestação (MAGALHÃES, 2011). Nessa perspectiva, a assistência a gestante deve 


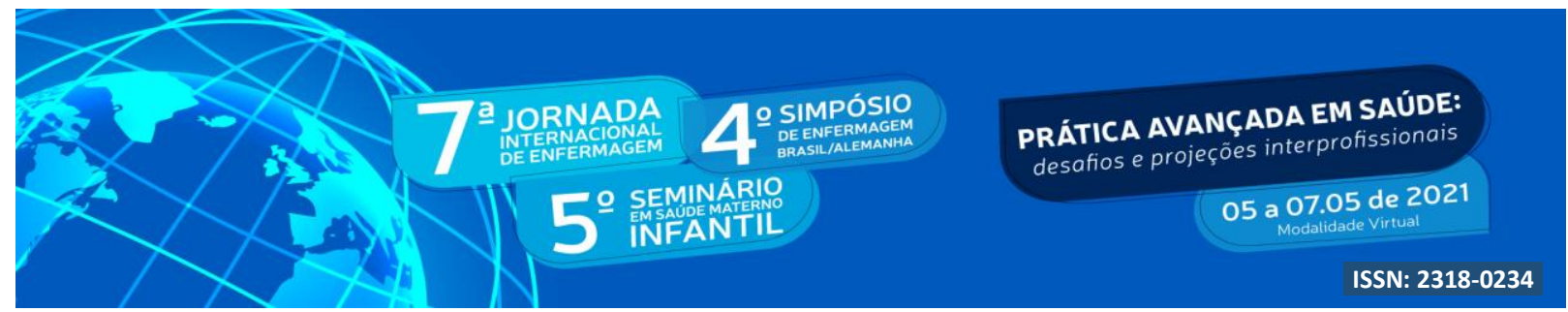

ter como principal objetivo evitar o comprometimento do feto e do recém-nascido, visando que todas as mulheres sejam assistidas de forma integral e adequadas às suas necessidades (MAGALHÃES, 2011).

Considerando este cenário, a EPS pode colaborar na atualização cotidiana das práticas, seguindo os novos aportes teóricos, metodológicos, científicos e tecnológicos, que contribuem para a construção de relações e processos, incluindo práticas interinstitucionais e intersetoriais. Desta maneira, constitui-se em um processo educativo que ocorre no âmbito do pensar e do fazer no trabalho, tendo como desafio a busca do estímulo para o desenvolvimento dos profissionais pautados em um contexto de responsabilidades e necessidades de atualização. A EPS é um processo compreendido como de reflexão e, a partir de ciclos de mudanças e transformações, pautados no serviço, no trabalho, no cuidado, inerente ao profissional da saúde, na educação e na qualidade da assistência (RICALDONI; SENA, 2006).

A sífilis ainda é um grave problema de saúde pública, mesmo sendo uma doença de fácil diagnóstico e tratamento. Assim, busca-se contribuir para a construção do planejamento de ações que possam diminuir os casos de sífilis na gestação e de sífilis congênita, por meio da EPS. Com isso, espera-se qualificar a assistência ao pré-natal, visando a integralidade das gestantes.

Para tal, é necessário um esforço conjunto na melhora da notificação compulsória dos casos, bem como na sua detecção e tratamento na assistência pré-natal. Além disso, devido ao seu impacto como doença causadora de inúmeras complicações e sequelas severas e irreversíveis em alguns casos, reforça-se a importância desta investigação, visando identificar os aspectos que podem ser melhorados.

Com essa leitura, visa-se possibilitar aos gestores em saúde, propor estratégias de ampliação de ações preventivas e diagnósticas que devem ser intensificadas no acompanhamento da gestante com sífilis, por meio da EPS com atividades como captação das gestantes, início precoce do pré-natal, identificação e notificação dos casos, busca ativa das faltosas e parceiro, entre outras. Ainda, sinaliza-se para o fomento de ações de educação em saúde sobre a doença, prevenção, transmissão e tratamento adequado na gestação, evitando a transmissão vertical e comprometimento fetal. 


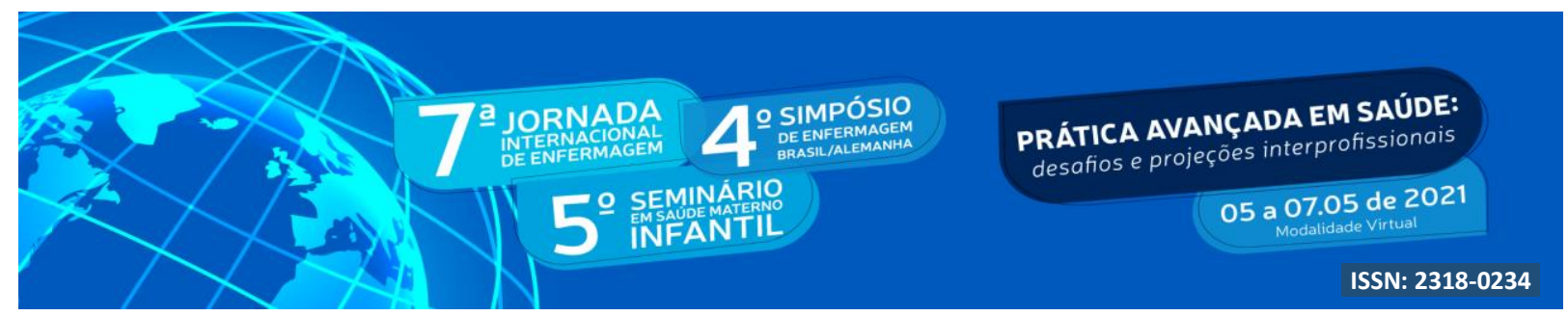

\section{OBJETIVO}

Qualificar os trabalhadores de uma Unidade de Saúde de um município do interior do Rio Grande do Sul sobre sífilis gestacional e congênita.

\section{MÉTODO}

Trata-se de uma proposta de intervenção a ser realizada em uma Unidade de Saúde de um município do interior do Rio Grande do Sul. Para isso, propõe-se a realização de dois encontros com os trabalhadores a seguir: 03 Médicos; 02 Odontólogos; 02 Enfermeiros; 02 Farmacêuticos; 01 Psicólogo; 01 Fonoaudiólogo; 02 Fisioterapeuta; 02 Técnicos de enfermagem; 02 Auxiliares de enfermagem; 01 Auxiliar de consultório dentário; 01 Agente de vigilância em saúde e 06 ACS. A proposta de intervenção será encaminhada para a aprovação da Secretária de Saúde, uma vez que o município não possui um órgão responsável pela EPS. Os encontros serão conduzidos pelo pesquisador em dia e horário a ser acordado com os participantes.

A proposta de intervenção abordará temáticas relacionadas ao fluxograma de acompanhamento dos casos de sífilis gestacional e congênita e a elaboração de um protocolo municipal sobre o tema. Destaca-se que a metodologia da intervenção acontecerá a partir das demandas já levantadas pela equipe após contato prévio com eles, na qual será apresentada a proposta e explicado sobre a importância da discussão, reflexão e ação de cada proposta. Nesse sentido, se buscará a participação ativa dos trabalhadores, de modo a fomentar a criação de estratégias que possam possibilitar pensar em dispositivos para a redução do número de casos de sífilis gestacional e congênita, fortalecendo a rede de atenção à saúde.

\section{RESULTADOS ESPERADOS}

Espera-se com a intervenção ampliar a cobertura de detecção precoce de sífilis gestacional e congênita, mediante a captação das gestantes e parceiro para a realização do 


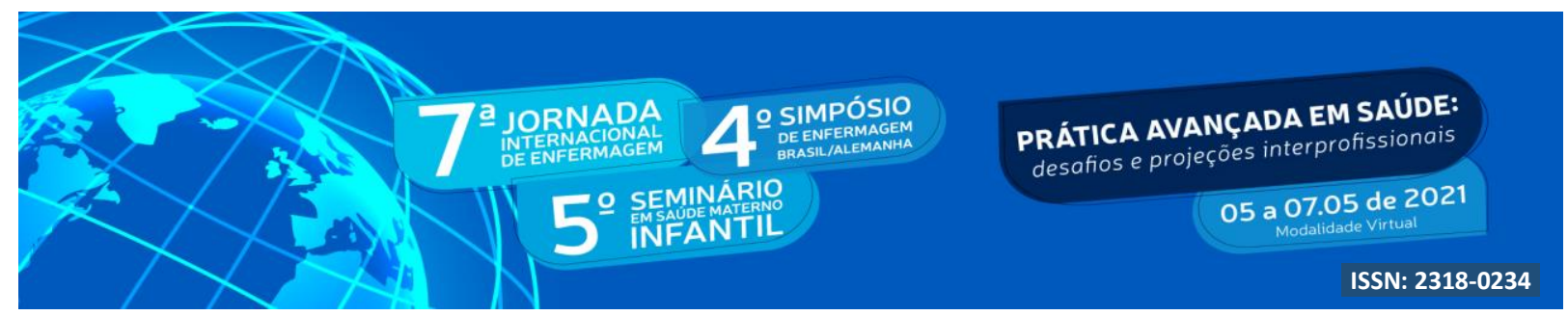

tratamento durante o pré-natal. Ainda, melhorar os registros das informações, a partir da capacitação de toda a equipe para o preenchimento correto da carteira da gestante e do seu prontuário, principalmente no que se refere ao tratamento realizado, destacando-se a sua importância para o planejamento em saúde e a qualificação do pré-natal realizado na Unidade. Assim, essas ações serão monitoradas a partir das coletas de informações constantes na carteira e prontuário das gestantes. Esses registros serão analisados pelo enfermeiro, no intuito de gerar dados e indicadores que serão discutidos com a equipe de saúde para qualificar a prática profissional e avaliar as ações desenvolvidas.

Os trabalhadores de saúde serão capacitados a partir de orientações que deverão ser fornecidas as gestantes durante o seu atendimento. Dessa forma, ressalta-se a importância da veiculação de informações necessárias para o cuidado. O fluxograma dos casos de sífilis gestacional e congênita será impresso e ficará disponibilizado em cada uma das salas de atendimento para consulta dos trabalhadores. A partir desse fluxograma será realizada a atualização dos trabalhadores, destacando-se o papel de cada profissional nas ações propostas. O protocolo do Ministério da Saúde servirá de base para a elaboração do protocolo municipal sobre o tema, o qual será construído posteriormente e, após, será impresso e disponibilizado para consulta dos trabalhadores de saúde. Ainda, nesse momento serão definidos quem fará o monitoramento dos indicadores e registros.

Para potencializar o processo de trabalho será criado um registro próprio que toda a equipe deverá ter conhecimento e acesso. Trata-se de fichas espelho que deverão ser preenchidas no momento do atendimento e que servirão de base para o levantamento de dados dos casos atendidos. Para atingir as metas e os indicadores propostos as gestantes e os parceiros serão monitorados e acompanhados pela ferramenta de registro. Para isso, será criado também um caderno de registros das gestantes que precisarão ser encaminhadas para o serviço de alto risco.

As ações desenvolvidas durante a intervenção visam garantir uma prática clínica de atenção à saúde integral. Assim, a intervenção proposta se tornará relevante para a equipe de saúde a partir da organização do seu processo de trabalho, fator importante para que as ações possam ser concretizadas e contribuam para a qualificação da assistência à gestante, por meio do aumento dos indicadores de saúde resultantes da intervenção. 


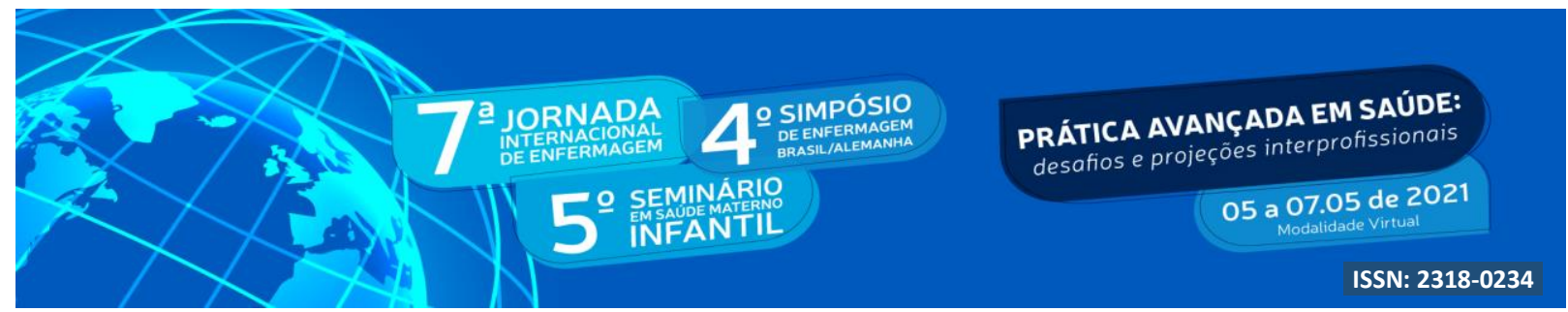

Também, a intervenção possibilitará sensibilizar a equipe de saúde para adequar as ações de melhoria e monitoramento do serviço. O trabalho propiciará refletir acerca do processo de trabalho desenvolvido na Unidade e a partir disso, promover mudanças nas ações para a melhoria do cuidado as gestantes. Além disso, será possível identificar as facilidades e as dificuldades que surgirão no decorrer do processo de implantação da intervenção.

Para a equipe, a intervenção proporcionará estreitar o vínculo com a gestante e para o serviço de saúde, possibilitará a melhoria nos indicadores de saúde. Dessa forma, espera-se que o registro das informações e o monitoramento sejam eficazes e as ações resolutivas. No que se refere à comunidade, se buscará a ampliação da satisfação das gestantes, por meio da melhoria na assistência e das ações realizadas no pré-natal, as quais são voltadas para a cuidado integral e humanizado.

Esta intervenção requer que as novas ações em saúde e a ampliação dos indicadores sejam discutidas com a comunidade, equipe de saúde e gestores, por meio da criação de espaços de diálogo. Salienta-se que os encontros propostos para a intervenção serão um desafio, mas espera-se que os resultados sejam satisfatórios, mostrando que várias ações podem ser melhoradas em relação à assistência à saúde da mulher.

\section{CONCLUSÃO}

A elaboração desta intervenção oportunizou refletir acerca da assistência à saúde da mulher, por meio da aproximação e abertura da equipe de saúde para a temática da sífilis. Soma-se a isso, a possibilidade de reflexão sobre a assistência realizada às gestantes, bem como o crescimento profissional e os futuros aprimoramentos nas ações de saúde que podem emergir deste processo em conjunto com a equipe de saúde.

Também foi possível aperfeiçoar o conhecimento a partir de uma proposta de intervenção que pode qualificar o processo de trabalho e de educação na saúde para o prénatal, buscando melhorias na assistência às gestantes atendidas na Unidade de Saúde e dos indicadores e ações que serão incorporadas no processo de trabalho da equipe de saúde.

\section{REFERÊNCIAS}




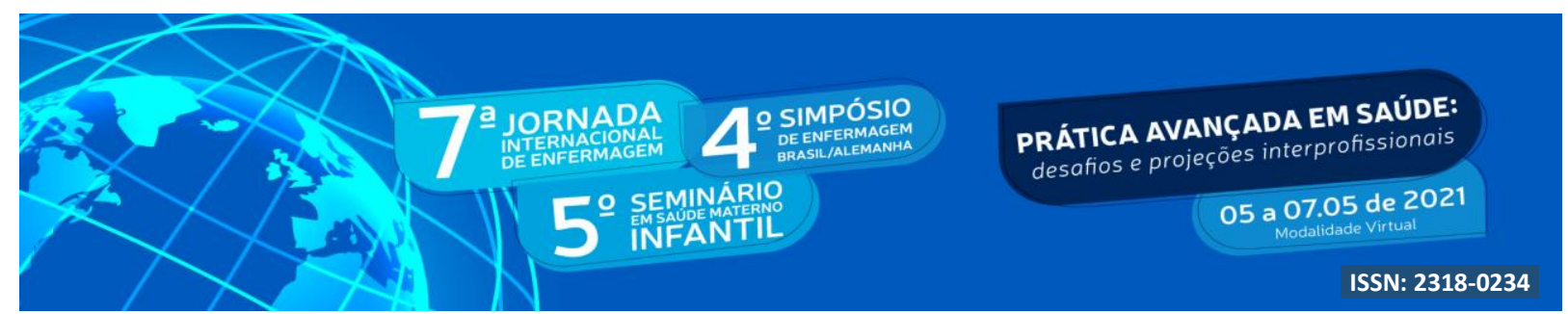

BRASIL. Ministério da Saúde. Política Nacional de Atenção Integral à Saúde da Mulher.

2004. Disponível em:

http://bvsms.saude.gov.br/bvs/publicacoes/politica_nac_atencao_mulher.pdf. Acesso em: 11 mar. 2021.

DAMASCENO, A. et al. Sífilis na gravidez. Revista Hospital Universitário Pedro Ernesto (HUPE), v. 13, n. 3, 2014.

DE LORENZI, DRS. et al. Transmissão vertical da sífilis: prevenção, diagnóstico e tratamento. Fêmina, v. 37, n. 2, p. 83-90, 2009.

MAGALHÃES, D.M.S et al. A sífilis na gestação e sua influência na morbimortalidade materno-infantil. Com. Ciências Saúde, v. 22, Sup.1, p. 43-54, 2011.

RICALDONI, C. A. C.; SENA, R. R. Educação permanente: uma ferramenta para pensar e agir no trabalho de enfermagem. Rev. Latino-am Enfermagem, Ribeirão Preto, v. 14, n.6, p. 837-842, nov.- dez., 2006.

VASCONCELOS, M.I.O. Sífilis na gestação: estratégias e desafios dos enfermeiros da atenção básica para o tratamento simultâneo do casal. Rev Bras Promoção Saúde, Fortaleza, 29(Supl): 85-92, dez., 2016. 